\title{
A influência da aerocinetose na aprendizagem e instrução aérea de cadetes aviadores da Força Aérea Brasileira
}

\author{
The influence of airsickness on learning and flight instruction \\ of aviator cadets of the Brazilian Air Force
}

\author{
Thiago Augusto Rochetti Bezerra¹, Leonel de Azevedo Neto², Fábio Angioluci Diniz Campos³ \\ $2^{\text {nd }}$ Lieutenant, Higher Education Teaching of the Brazilian Air Force. Estrada de Aguai s/n. Pirassununga, SP, Brasil. Post-Doctoral researcher at the Institute \\ of Physics of São Carlos/USP. Institute of Physics of São Carlos. <thiago_rochetti@globomail.com> \\ 2 Cadet Airman of the $4^{\text {th }}$ Squadron of the Air Force Academy < < leonel.azevedo azv@hotmail.com>. \\ ${ }^{3} 2^{\text {nd }}$ Lieutenant, Higher Education Teaching of the Brazilian Air Force. Pirassununga, SP, Brasil. Doctoral candidate in Human Movement Sciences, Methodist University \\ of Piracicaba. Estrada de Aguai s/n. Pirassununga, SP, Brasil.<fabiocampos06@gmail.com>. \\ Study conducted at the Air Force Academy, AFA, Pirassununga, SP, Brazil.
}

\begin{abstract}
RESUMO
A Aerocinetose é uma resposta fisiológica normal à percepção de movimento não usual, ocorrendo frequentemente em aviadores. $\mathrm{O}$ objetivo desta pesquisa foi investigar a prevalência de aerocinetose entre cadetes aviadores da Aeronáutica brasileira em Pirassununga, estado de São Paulo, sua busca por tratamento médico e a influência dos sintomas no seu desempenho. Para tanto, foi realizado um estudo transversal de prevalência, baseado em um questionário criado pelos pesquisadores. Os cadetes responderam individualmente a 8 perguntas objetivas. O número de cadetes avaliados foi de 186 . A idade média foi de $(22,5 \pm 1,8)$ anos, sendo a maioria do sexo masculino $n=174$. O pequeno número de mulheres não permite inferir comparação entre os sexos. O número de cadetes com diagnóstico de aerocinetose foi $112(60,2 \%)$, enquanto que em estudos internacionais varia de $10 \%$ a $39 \%$. A fase de manobras e acrobacias foi apontada com maior incidência de aerocinetose, citadas por 75 dos 186 cadetes. Dos 112 cadetes diagnosticados com aerocinetose, 37 relataram que seu desempenho foi afetado em uma missão, e dos cadetes que apresentaram sintomas, 91\% procuraram assistência médica após a crise. Entre os que já se submeteram a algum tratamento $(\mathrm{n}=102), 98$ cadetes utilizaram medicamento, 58 relataram exercícios de cama elástica prescritos pelo médico e 2 fizeram tratamento através da acupuntura. Apenas 35 cadetes relataram que houve melhora após algum tipo de intervenção. Entre os cadetes brasileiros há uma incidência maior que a encontrada nos estudos internacionais. A maioria deles não realizou tratamento especializado, apesar de seu desempenho ser prejudicado durante sua rotina de voos.

PALAVRAS-CHAVE: Cinetose. Militares. Enjoo em voo. Prevalência.
\end{abstract}

\section{ABSTRACT}

Airsickness is a normal physiological response to the perception of unusual motion, often occurring in aviators. This research aimed to investigate the prevalence of airsickness among aviator cadets of the Brazilian Air Force,

Pontifical Catholic University of Rio Grande do Sul Porto Alegre, RS, Brazil

Editor

Thaís Russomano

Microgravity Centre PUCRS, Brazil

Executive Editor

Rafael Reimann Baptista

Faculdade de Educação Física e Ciências do Desporto, PUCRS, Brazil

e-ISSN: 2179-703X

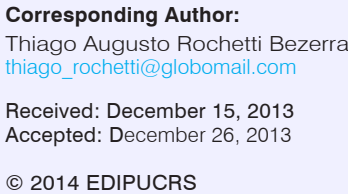

(c) 2014 EDIPUCRS

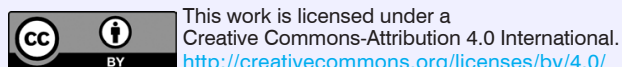


based in Pirassununga, São Paulo state, their search for medical treatment and the influence of symptoms on performance. A cross-sectional study of prevalence was conducted, based on a questionnaire created by the researchers. Cadets responded individually to 8 objective questions. The number of cadets evaluated was 186 , with a mean age $( \pm S D)$ of $22.5( \pm 1.8)$ years, and being mostly male $(n=174)$. The small female sample did not allow comparison between genders. Cadets affected by airsickness numbered 112 (60.2\%), with this figure ranging from $10 \%$ to $39 \%$ in international studies. The maneuvers and acrobatics phase was identified with the higher incidence of airsickness, cited by 75 of the 186 cadets. Of the 112 cadets diagnosed with airsickness, 37 reported their mission performance being affected, and of those cadets presenting symptoms, $91 \%$ sought medical assistance after the crisis. Among those already having undergone some treatment $(n=102), 98$ cadets used medication, 58 reported trampoline exercises prescribed by a doctor and two were treated with acupuncture. Only 35 cadets reported an improvement after some form of intervention. A higher incidence was encountered among the Brazilian cadets than found in international studies. Most of those affected did not carry out specialized treatment, even though their performance was impaired during their flight routine.

KEYWORDS: Motion sickness; Military personnel; Airsickness; Prevalence

\section{Introdução}

Os cadetes aviadores que estão cursando o segundo ano do curso de formação de Oficiais Aviadores da Forca Aérea Brasileira (CFOav), realizam um curso básico de pilotagem militar no Segundo Esquadrão de Instrução Aérea (2ํㅡㄹ EIA) com a aeronave T-25 UNIVERSAL. Nesta fase de aprendizagem ao voo, os cadetes desenvolvem qualidades individuais de pilotos militares. As fases de aprendizagem de voo são; manobras e acrobacias (MAC), voos de formatura e navegações. Durante os voos, alguns cadetes enfrentam um problema relacionado ao fato de sentir sintomas ocasionados por movimentações da aeronave, essa movimentação afeta os sistemas responsáveis por manter o equilíbrio do corpo humano (sistema vestibular, visão e sistema proprioceptivo). Em alguns casos, estes sintomas acarretam prejuízos na instrução Aérea.

\subsection{Aerocinetose}

A cinetose é um distúrbio funcional que é desencadeado por movimentos e acelerações bruscas e estimulação excessiva do aparelho vestibular. A cinetose é caracterizada por diversos sintomas, como por exemplo, náusea e vômito. Os impulsos nervosos passam da orelha interna para o centro do bulbo, ocasionando náuseas e vômitos (Tortora, 2000).

A aerocinetose consiste em uma resposta fisiológica normal universal à percepção de movimento não usual, podendo ocorrer como reação à exposição ao voo. A patogênese da cinetose não é completamente compreendida, mas a explicação mais aceita atualmente é a teoria do conflito sensorial (Schmäl, 2013; Shupak e Gordon, 2006).

\subsection{Efeitos da Aerocinetose na formação do Cadete Aviador}

Durante o segundo ano do curso (CFOav), após sedimentados os conhecimentos sobre a estrutura da aeronave, procedimentos a serem realizados durante o voo e como lidar com panes durante as missões militares de voo, o cadete começa a entrar em contato com a aprendizagem prática da aviação.

O cadete que sofre com a aerocinetose, além de se preocupar com os fatores intrínsecos (manuseio da aeronave) e extrínsecos (condições ambientais) que estão presentes no voo, deve se manter concentrado para os sintomas não afetem seu desempenho na missão, tornando o voo por ele realizado deficiente.

\subsection{Formação do Aviador na Força Aérea Brasileira (FAB)}

O currículo mínimo do CFOAV consta de uma carga horária total de curso de 8.800 horas, distribuídas em 4 anos e em 220 dias letivos por ano. Essa carga horária ainda está subdividida entre os diversos campos que deverão ser cumpridos ao longo do curso; assim, o campo Geral dispõe de 3.593 horas de aula; o campo Técnico-Especializado de 1.936 horas de aula; o campo Militar de 1.794 horas de aula e as Atividades Administrativas de 1.477 horas de aula.

O curso de formação de Oficiais Aviadores (CFOAV) é reconhecido pelo Ministério da Educação como curso superior (Parecer no 672/80), que tem por objetivo a formação do Oficial Aviador e está estruturado em uma única fase. A duração é de 4 (quatro) anos em que são ministradas instruções Gerais, Técnico-Especializado e Militar (2 IA, 2012). 
Durante o primeiro ano na academia, os Cadetes cursam disciplinas que são comuns aos três cursos oferecidos pela AFA: o Curso de Oficiais Aviadores, o Curso de Oficiais Intendentes (CFOINT) e o Curso de Oficiais de Infantaria (CFOINF).

No primeiro ano, os aviadores fazem disciplinas técnico-especializado (Aerodinâmica, Instrução Técnica e Navegação Aérea).

A atividade aérea, discriminada no campo TécnicoEspecializado, dispõe da seguinte carga horária: Instrução de Voo na aeronave T-25 (Figura 1), que ocorre no segundo ano, 522 horas de aula; sendo que este tempo esta dividida entre aulas práticas e teóricas.

No terceiro ano, ou 3ํㅡ esquadrão, os cadetes voltam a cumprir basicamente disciplinas do campo geral. Os mesmo prosseguem com a rotina diária, com os treinamentos físicos e militares, e cumprem novamente algumas disciplinas do campo técnico-especializado (Aerodinâmica 2 e Instrução Técnica da Aeronave T-27) que será pré-requisito para o voo na aeronave T-27 (Figura 2), para a qual deverão estar teoricamente preparados no início do quarto ano.

Figura 1. Aeronave T-25 Universal Texas ${ }^{\circledR}$

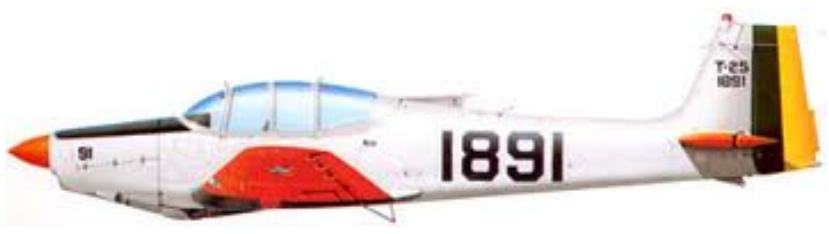

Fonte: AFA.

Figura 2. Aeronave EMB 312 T-27

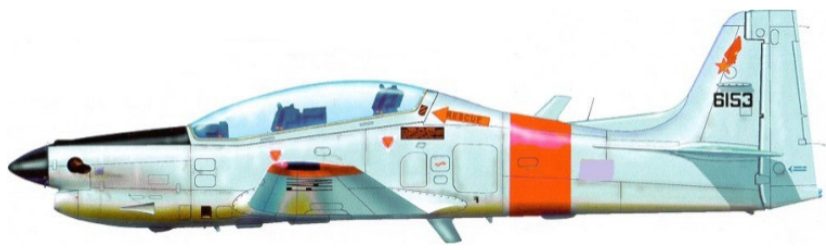

Fonte: AFA

No último ano do curso os cadetes do CFOAV retomam o voo no $1^{\mathrm{O}}$ EIA, voando na aeronave $\mathrm{T}-27$ "Tucano".

\subsection{Aeronaves de Instrução da Academia da Força Aérea (AFA)}

São utilizados pela AFA dois modelos de aeronaves de instrução aérea. No segundo ano são utilizadas as aeronaves $\mathrm{T}-25$, e no quarto ano as aeronaves T-27.
A aeronave T-25 Universal é utilizada para a instrução básica, pois permite que o instrutor permaneça sentado ao lado do cadete, o que na fase inicial é aconselhável. Outro motivo de ser o T-25 usado para a instrução do segundo ano é sua facilidade de comando quando comparado com o T-27. A velocidade máxima atingida é de $275 \mathrm{~km} / \mathrm{h}$, sendo que a força $\mathrm{Gz}$ atingida por essa aeronave é também menor.

A aeronave EMB 312 T-27 é um turbo hélice que inovou o mercado no início da década de oitenta, ao introduzir, entre outras novidades, assentos ejetáveis Martin Baker BR8LC ${ }^{\circledR}$ (Manual EMB, 1984).

A força $\mathrm{Gz}+$ máxima atingida pela aeronave EMB $312 \mathrm{~T}-27$ é de $6 \mathrm{Gz}$ (positivo) e $3 \mathrm{Gz}$ (negativo) em situação de voo invertido, sendo que neste ultimo caso a aeronave pode permanecer de dorso em um tempo máximo de 30 segundos (Manual EMB, 1984).

\subsection{Etapas de aprendizagem de voo}

A aprendizagem de voo é dividida em quatro etapas: voo solo, manobras e acrobacias, voo de formatura e navegação. Estas quatro fases são realizadas pelos cadetes do $2^{\mathrm{O}}$ e $4^{\mathrm{O}}$ anos variando o tipo de aeronave e o número de missões (2ํㅡㄹ EIA, 2012).

\subsubsection{Voo Solo}

Esta é uma etapa primordial na formação do Aviador. O Cadete deverá nesta fase ser capaz de decolar e pousar a aeronave. Após uma sequência de aulas acompanhado de um Instrutor do $2^{\circ} \mathrm{EIA}$, o Cadete realiza pela primeira vez o voo solo. Neste voo o Cadete recebe uma rota definida, e deverá decolar sozinho com a aeronave. Após realizar a rota estabelecida, o Cadete retorna com a aeronave para o pouso. Nesta primeira fase muitos Cadetes são desligados por apresentarem deficiências na aprendizagem e execução.

\subsubsection{Manobras e Acrobacias (MAC)}

A fase de manobras e acrobacias é a segunda etapa de formação do aviador. Nesta etapa o instrutor realiza várias acrobacias com o Cadete, tais como o looping, touneau lento e barril, returnemant, meio oito cubano, estol, entre outras.

Todo o conhecimento do Cadete quanto à movimentação do manche (manobras), com os movimentos de Cabrar, Picar, Rotação para direita e esquerda, já aprendidas na fase solo, é utilizado nesta fase mais complexa.

Esta etapa é complicada no aspecto fisiológico, pois a exposição à força $\mathrm{Gz}+$ leva a alguns Cadetes 
sentirem mal, ter enjoos e dores de cabeça. Há casos de Cadetes sofrerem de distúrbios, levando ao desmaio. A incapacidade em suportar a força $\mathrm{Gz}+$ leva ao desligamento do Cadete do curso de aviação.

A Figura 3 demonstra a uma aeronave T-27 realizando manobra de uma rotação para a esquerda (esquerda da foto), enquanto que a outra aeronave permanece em voo reto nivelado (direita da foto).

\subsubsection{Etapa de Formatura}

Depois de familiarizados com o voo solo e manobras e acrobacias o Cadete se prepara para a terceira etapa. Este voo e realizado com 6 a 8 aeronaves perfiladas (Figura 4), sendo que a distância entre aeronaves deve ser de aproximadamente 3 metros (2 EIA, 2012).

\subsubsection{Navegação}

A etapa de Navegaçãoé de fundamental importância para a experiência do piloto. Nesta etapa o Cadete se prepara para solucionar panes internas à aeronave, tais como corte de combustível, pane elétrica, pane de motor, fogo na aeronave, e intercorrências externas, como acidentes com aves, tempestades, ventos fortes entre outras situações. Nesta etapa de treinamento é bastante utilizado o simulador da aeronave T-27 (Figura 5). Este equipamento pode simular todas estas situações de pane através de um painel de controle (2 EIA, 2012).

Figura 3. Manobra de Rotação para esquerda com Aeronave EMB 312 T-27 ${ }^{\circledR}$

Figura 4. Fase de Formatura

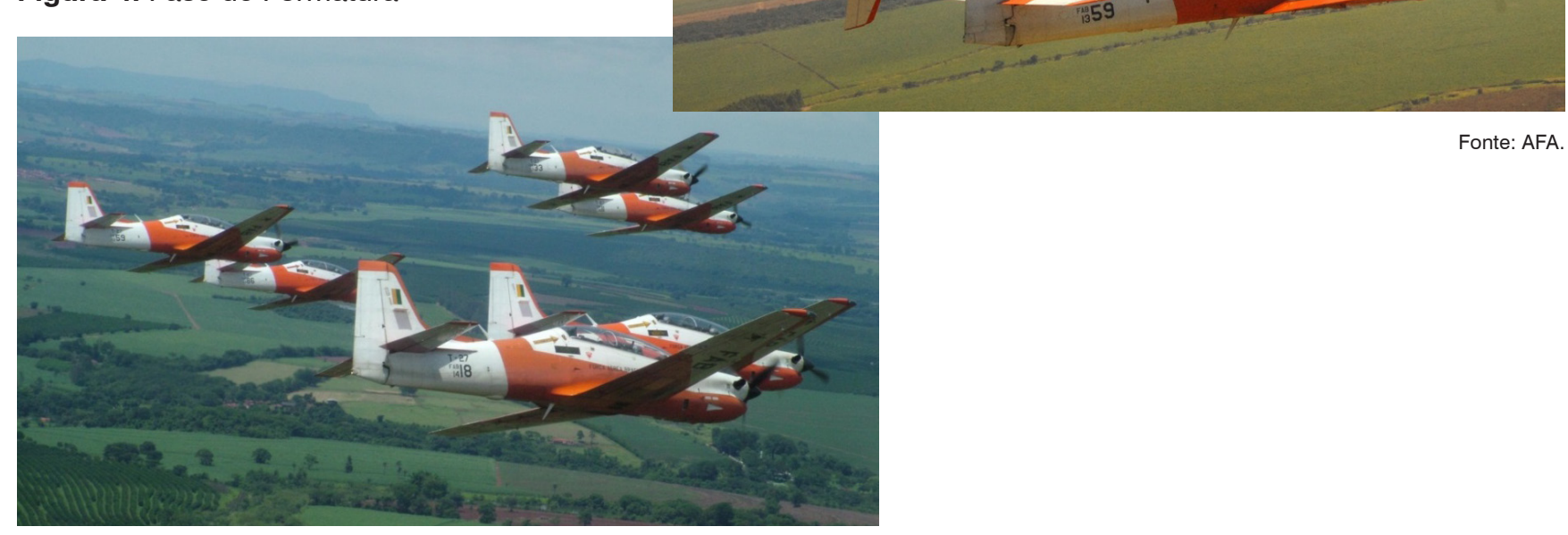

Fonte: AFA.

Figura 5. Sala de comando do Simulador de Voo da aeronave T-27
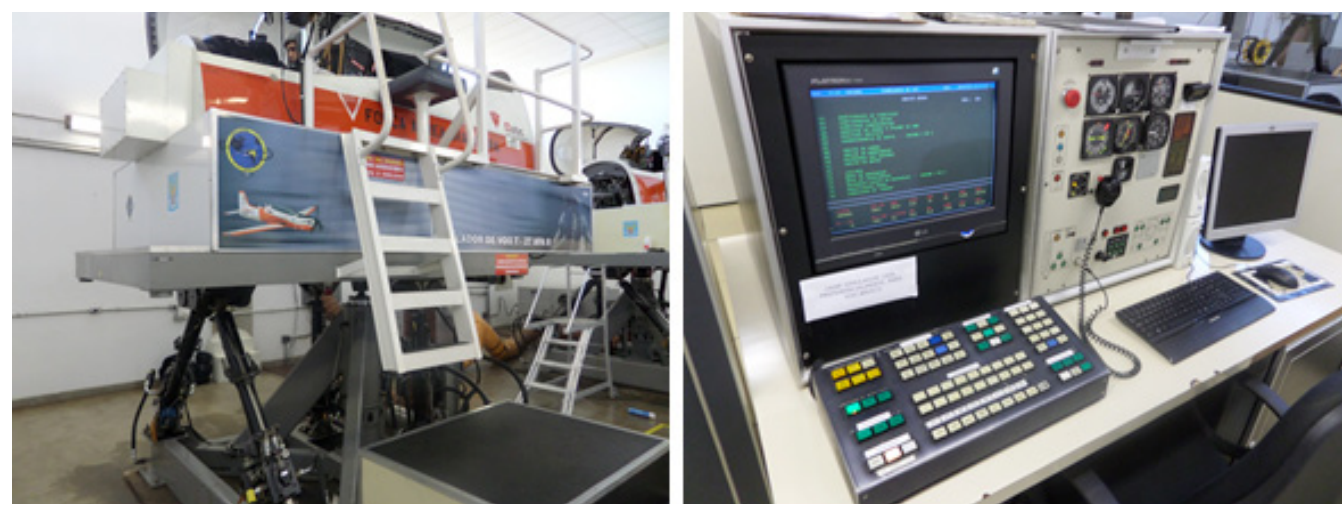

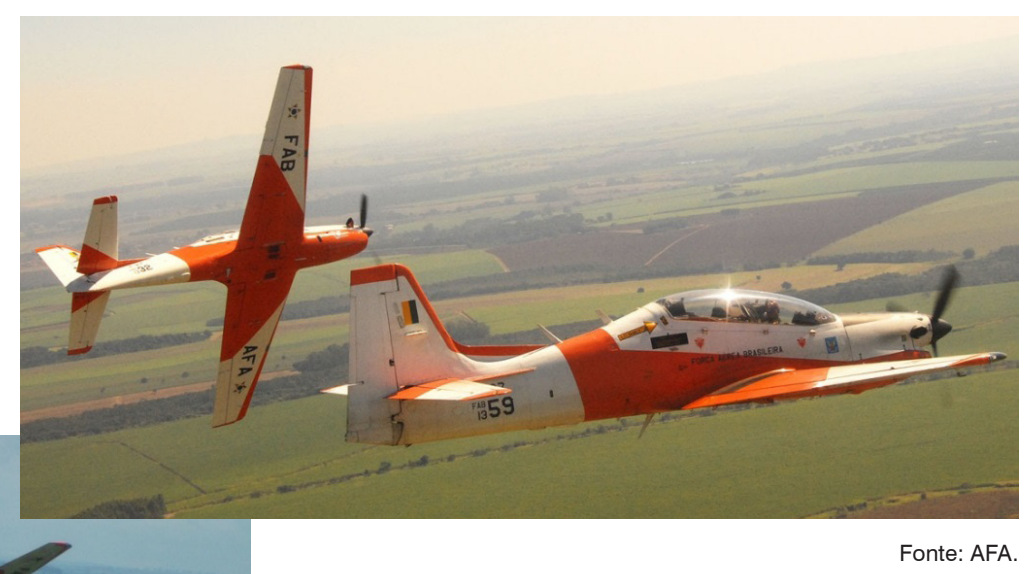


O Cadete deverá prontamente solucioná-las. Após horas no simulador, ocorre um voo simulado de navegação, geralmente utilizando-se de rotas por bases aéreas por todo o Brasil. Uma rota bem comum se faz entre a base aérea de Pirassununga-SP e as de Natal- RN e Manaus-AM (2을 EIA, 2012).

\section{Objetivos}

- Identificar a prevalência de sintomas de Aerocinetose entre os cadetes em treinamento de aviação da Força Aérea Brasileira.

- Verificar a incidência dos cadetes acometidos por aerocinetose.

- Verificar os sintomas que causam impacto na aprendizagem de voo.

\section{Metodologia}

A presente pesquisa teve como grupo experimental os cadetes Aviadores da Força Aérea Brasileira (FAB). Caracterizou-se por uma investigação epidemiológica de natureza quantitativa e de cunho exploratório, ao verificar a prevalência de aerocinetose em cadetes aviadores da FAB.

Foi aplicado um questionário com 8 (oito) questões acerca da incidência do efeito da aerocinetose no voo sobre a saúde dos cadetes, relacionadas ao objetivo do estudo.

Os dados foram analisados de forma quantitativa, por meio de medidas descritivas, sendo resguardadas a privacidade de cada avaliado e a confidencialidade dos dados obtidos.

\subsection{Questionário}

O questionário foi elaborado pelos pesquisadores.

Foram realizadas questões objetivas, para investigar a existência de sintomas característicos da cinetose e de fatores associados aos possíveis episódios, como a interferência no desempenho durante o voo e a realização ou não de tratamento. As perguntas foram as seguintes:

1) Ao longo dos vôos no $2^{\mathrm{o}}$ EIA, você sofreu algum efeito da aerocinetose?

$$
\text { Sim ( ) Não ( ) }
$$

2) Quais sintomas da aerocinetose você teve ao longo dos vôos?

$\begin{array}{ll}\text { Sudorese ( ) } & \text { Enjoo ( ) } \\ \text { Bocejos ( ) } & \text { Vômito ( ) } \\ \text { Salivação ( ) } & \text { Outros ( ) }\end{array}$

3) Em qual fase do vôo iniciou-se os sintomas da aerocinetose?

Pré-solo ( ) Formatura ( )
Manobras e Acrobacias (MAC) ( )
Navegação ( )

4) Teve a instrução prejudicada após sofrer a ação da aerocinetose?

Sim ( ) Não ( )

5) Teve o vôo abortado pelo fato de não conseguir prosseguir na missão?
Sim ( )
Não ( )

6) Quando descobriu que sofria da aerocinetose, procurou a médica do esquadrão em busca de algum tratamento?

Sim ( ) Não ( )

7) $\mathrm{O}$ que você procurou fazer para amenizar os efeitos da aerocinetose?

Acupuntura ( ) Medicamentos ( )
Outras ( )
Cama elástica ( )
Nenhuma medida tomada ( )

8) As medidas adotadas para amenizar o efeito da aerocinetose contribuíram para que houvesse alguma melhora?

Sim ( ) Não ( )

\section{Resultados}

\subsection{Perfil da população estudada}

O número de indivíduos que responderam ao questionário foi de 186 cadetes, sendo 12 do gênero feminino e os demais do gênero masculino. A média de idade foi de $(22,5 \pm 1,8)$ anos. A média de instrução em aulas praticas de voo foram de 86,15 horas.

\subsection{Prevalência e sintomas da Aerocinetose na população estudada}

Dos 186 indivíduos, 112 apresentaram diagnóstico de aerocinetose (60,2\% do total de entrevistados). Entre os cadetes que apresentaram aerocinetose, $21(18,7 \%)$ apresentaram sudorese em excesso, 3 (2,6\%) bocejos, $5(4,4 \%)$ salivação excessiva, $82(73,2 \%)$ enjoo, 95 $(84,8 \%)$ vômito, e 5 (4,4\%) apresentaram outros sintomas (Gráfico 1). 


\section{Gráfico 1. Sintomas da aerocinetose em cadetes}

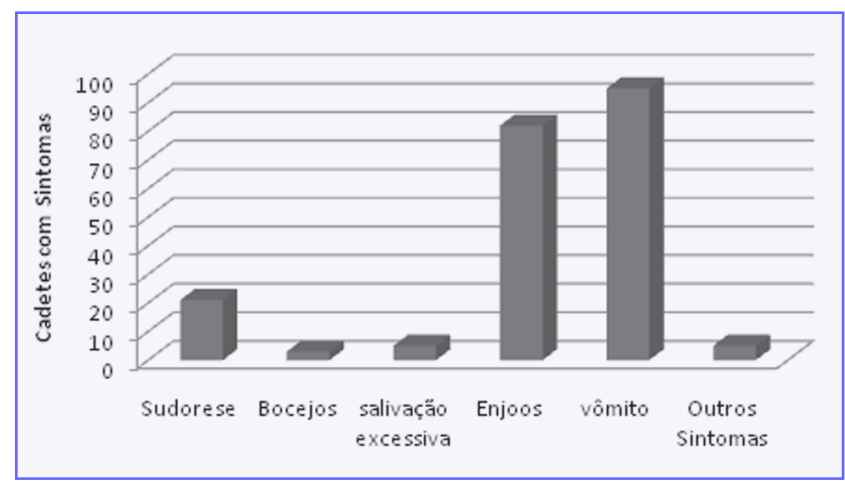

\subsection{Interferência da Aerocinetose no desempenho da aeronavegação}

Dos cadetes que apresentaram aerocinetose $(n=112), 56(50 \%)$ apresentaram os sintomas na fase solo e Pré-solo, 11 (10,1\%) na fase de formatura, 75 $(66,9 \%)$ na fase Manobras e Acrobacias (MAC), e apenas 2 cadetes $(1,7 \%)$ na fase de navegação (Gráfico 2). Destes $68(60,7 \%)$ cadetes relataram ter a instrução prejudicada pela ação da aerocinetose, e 37 (33\%) abortaram a missão devido aos sintomas.

\section{Gráfico 2. Incidência da Aerocinetose nas fases de aprendizagem aérea}

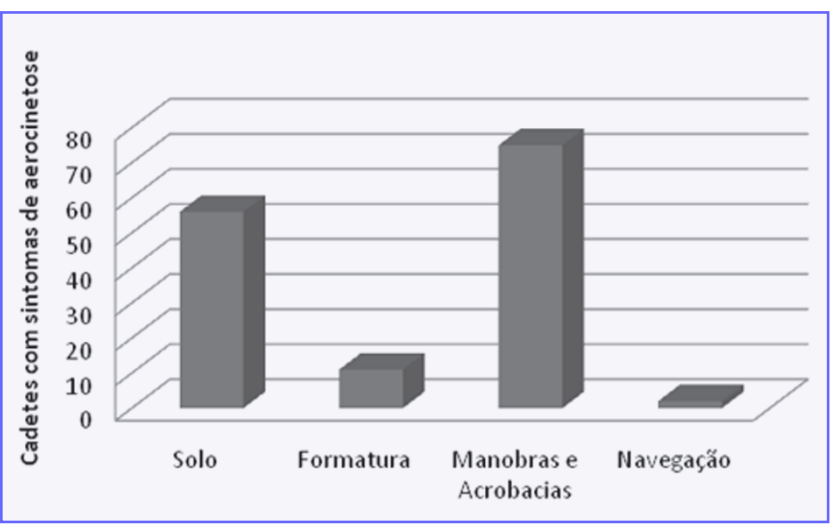

\subsection{Aerocinetose e a busca por tratamento}

Dentre os cadetes que relataram quadro de aerocinetose $(n=112), 102 \quad(91 \%)$ procuraram assistência médica após a crise. Entre os que já se submeteram a algum tratamento $(\mathrm{n}=102), 98$ cadetes utilizaram medicamento, 58 relataram exercícios de cama elástica prescritos pelo médico e dois fizeram uso de outros tipos de tratamento através da acupuntura.
Apenas 35 cadetes relataram que houve melhora após algum tipo de intervenção.

\section{Discussão}

Verificou-se, na população estudada, uma prevalência de 60,2\% de aerocinetose entre os 186 cadetes aviadores da Academia da Força Aérea Brasileira, em Pirassununga, estado de São Paulo, porcentagem ligeiramente superior à encontrada na literatura internacional, que varia de $10 \%$ a $39 \%$ entre estudantes de aviação militar (Lucertini et al., 2008; Giles; Lochridge, 1985; Benson; Tott; 2006; Rashedin et al., 2009). Entretanto, segundo dados da National Aeronautics and Space Administration (NASA), acinetose acomete aproximadamente 50\% dos aviadores militares em algum momento da sua carreira (Acromite, 2011).

Percebe-se uma quantidade bem reduzida de cadetes do sexo feminino (apenas 12, entre 186 entrevistados). Isso pode ser devido ao ingresso recente das mulheres no quadro dos aviadores, que ocorre desde 2003. O número não foi suficiente para estabelecer relação entre os gêneros.

No estudo feito na Academia da Força Aérea de Bangladesh, 57\% dos estudantes militares que apresentam sintomas de aerocinetose desenvolveram durante a fase de MAC, índice menor que os , e 66,9\% na Academia da Força Aérea Brasileira(Rashedin et al., 2009).

Além desse impacto negativo nas atividades aéreas, a aerocinetose pode levar ao término prematuro do voo, afetar negativamente a motivação do piloto e gerar uma grande ansiedade.

Neste trabalho, foi marcante a adesão ao tratamento médico: 91\% dos 112 indivíduos acometidos por aerocinetose realizaram um tratamento.

A alta adesão pode ser parcialmente explicada pelo fato de, no ambiente militar este fator pode ser preponderante no sucesso e da continuidade dos estudos na AFA.

No entanto, devido a rotina intensa na AFA, muitos cadetes optam por medicamentos e não procuram tratamentos que dispendem maior tempo, tais como cama elástica e aculpultura.

A Medicina Aeroespacial poderia ser utilizada como medida educativa no primeiro ano do curso de Oficiais aviadores na Academia da Força Aérea. Em seguida realizar uma triagem e oferecer um tratamento preventivo, antes de se iniciarem as atividades aéreas. Isto poderia minimizar - ou até eliminar - os sintomas e aumenta aumentar a motivação do cadete, favorecendo o desenvolvimento maior de suas poten- 
cialidades. Desta forma, é altamente recomendável que se realize uma minuciosa busca pelos cadetes com sintomas de aerocinetose, preferencialmente antes de iniciarem suas atividades aéreas, para lhes oferecer esclarecimento e tratamento precoces dessa importante afecção.

\section{Considerações finais}

Este trabalho teve como objetivo investigar como a aerocinetose afeta desempenho dos cadetes aviadores durante os voos de instrução. Os dados obtidos na pesquisa indicam que os sintomas da aerocinetose acometem os cadetes com bastante frequência, além de impactarem negativamente o desempenho desses aprendizes durante as instruções de voo. As manifestações relatadas mais comuns foram o enjoo e vomito. A fase em que se verificou a maior incidência de aerocinetose foi de Manobras e Acrobacias.

Foi constatado que a aerocinetose gera desperdício de tempo e dinheiro, uma vez que muitos voos tiveram que ser interrompidos e refeitos em virtude de suas reações. A segurança do voo também fica prejudicada, uma vez que o cadete realiza voos solo.

Dessa forma, é necessário que se busque identificar e tratar os cadetes com maior suscetibilidade em apresentar a aerocinetose no ambiente aéreo antes do início das instruções aéreas. Tal medida contribuiria para a diminuição dos efeitos adversos que acarretam um desempenho inferior às capacidades dos aviadores militares aprendizes.

\section{Referências}

Acromite $\mathrm{M}$ et al. Operational applications of autogenic feedback training exercise as a treatment for airsickness in the military. In: WIML-NASA Workshop, 2011, Warsaw, Poland. [Anais...], Warsaw, Poland; 2011. p. 29.

Benson AJ, Stott JRR. Motion Sickness. In: Rainford DJ, Gradwell, D. P. (Ed.). Ernsting's aviation medicine. 4th ed. London: Hodder Arnold; 2006.

Giles DA, Lochridge GK. Behavioral airsickness management program for student pilots. Aviation, Space and Environmental Medicine (Alexandria) 1985; 56(10):991.

Gordon CR et al. The effects of dimenhydrinate, cinnarizine and transdermal scopolamine on performance. Journal of Psychopharmacology 2001; 15(3):167-72.

Lucertini $\mathrm{M}$ et al. Effects of airsickness in male and female student pilots: adaptation rates and 4-year outcomes. Aviation, Space and Environmental Medicine (Alexandria) 2008; 79(7): 677-84.

Manual EMB. Normas técnicas da Aeronave Embraer T-27. Rio de Janeiro: Força Aérea Brasileira; 1984.

Rashedin L et al. Motion sickness in student pilots of Bangladesh Air Force Academy: a study of 80 cases. Sleep 2009; 5:25.

Schmäl F. Neuronal mechanisms and treatment of motion sickness. Pharmacology 2013; 91(3-4):229-41.

Shupak A, Gordon CR. Motion sickness: advances in pathogenesis, prediction, prevention, and treatment. Aviation, Space and Environmental Medicine (Alexandria) 2006; 77 (12):1213-23.

2ํㅡㄹ Esquadrão de Instrução Aerea. Ministério da Defesa. Manual de Instruções da Aviação. Pirassununga, SP: Comando da Aeronáutica; 2012.

Correspondence address:

Thiago Augusto Rochetti Bezerra

Av. José Antunes de Lisboa, 840, apto 13

13613-130 Leme, SP, Brasil

Tel.: (019) 9976-78229

<thiago_rochetti@globomail.com> 